\title{
UV Emission and Spectral Synthesis of Accretion Disks in Non-Magnetic Cataclysmic Variables
}

\author{
Raul E. Puebla and Marcos P. Diaz \\ Departamento de Astronomia. IAG. Universidade de São Paulo. \\ Brazil
}

\section{Introduction}

Accretion disks are present throughout the wide Universe at multiple scales. They are in the active galactic nuclei $(\mathrm{AGN})$ with a disk radius $\left(r_{\text {disk }}\right) \sim 0.1-1.0$ parsecs $(1.0 \mathrm{pc} \sim 206264 \mathrm{AU}$ $\left.\sim 3.1 \times 10^{18} \mathrm{~cm}\right)$, protostars (YSOs) $\left(r_{\text {disk }} \sim 1000 \mathrm{AU}\right)$, Debris disks $\left(r_{\text {disk }} \sim 10-100 \mathrm{AU}\right)$ and in close binary systems: Cataclysmic Variables (CVs), Low Mass X-Ray Binaries (LMXBs) and some symbiotic stars $\left(r_{\text {disk }} \sim \mathrm{R}_{\odot} \sim 6.9 \times 10^{10} \mathrm{~cm}\right)$. Being a very common phenomenon in the Universe, the understanding of accretion disk behavior, time scales and evolution are crucial to have a clearer vision of most of the systems present in the Universe.

Among these systems, interacting binaries are the best laboratories to understand the accretion disk physics. This because of its abundance in the Galaxy, the amount of nearby systems, data availability and the existance of observational facilities to obtain them. Cataclysmic Variables present the best scenery to analyze accretion disk behavior due to the fact that its main emission region falls on ultraviolet (UV) and optical. In these wavelength bands, we have the tools with the spectral and the time resolution that allow us to see most of the disk with good detail. Also, due to the order of magnitude of CVs periods and luminosity, it is feasible to study the disk physics in a wide range of time scales, which facilities the understanding of its evolution in a multiplicity of cases.

Cataclysmic Variables are semidetached binary systems with orbital periods between 0.28 and 18 hours (Ritter \& Kolb, 2003), where a Roche-lobe filling main sequence star (secondary) transfers mass onto a white dwarf (primary). Depending on the magnitude of the primary magnetic field, this mass transfer can be performed through an accretion disk (non-magnetic CVs), a truncated disk (intermediate polars) or funnels (polars). CVs can also be classified into several types depending on their erupting behavior. System that do not show any eruptions is called "Nova - Like" (NL), systems that show one or more thermonuclear eruptions are "Novae" (Ne) and "RecurrentNovae" (RN) respectively. Also, there exists systems with a kind of "weak" periodic eruption, caused by disk instabilities. These systems are known as "Dwarf Novae" (DNe) (Warner, 1995). It is important to score that the types of CVs are not exclusive each other i.e. both magnetic or non-magnetic CVs can show novae eruptions.

The NL systems are non-magnetic CVs with a high mass accretion rate $\left(\dot{\mathrm{M}}_{a}\right)$. This fact bears a bright accretion disk, unlike DNe that show low $\dot{\mathrm{M}}_{a}$ values and then weaker disks. For NL the disk emission greatly exceeds the emission of the other members of the binary system (primary plus secondary), and specially dominates the UV region of the spectrum. Also, it has 
been set that high accretion rates through the disk guarantees its stability (see Lasota, 2001). Because of these reasons, NL are the best systems to study the emission, origin and physics of disk radiation as well as to limit the disk models. Furthermore, due to the strong dependence of the UV spectra on the accretion rate, white dwarf mass $\left(\mathrm{M}_{\mathrm{WD}}\right)$ and disk radius $\left(r_{\text {disk }}\right)$, UV spectroscopy is a crucial tool to establish the binary system parameters, and to find the correlation between them. Likewise, these correlations could help to constrain the evolution models for these kind of close binary systems (see Rappaport et al., 1982; 1983).

In this chapter we expose the different efforts made through the years to reproduce the UV spectral observations, using several methods of spectral synthesis. Also, we focus on different tests done on those different methods through UV data taken from the different satellites as International Ultraviolet Explorer (IUE), Hubble Space Telescope (HST), Hopkins Ultraviolet Telescope (HUT) and Far Ultraviolet Spectroscopic Explorer (FUSE). In recent years important advances have been done in the sense of building a consistent model of accretion disk plus wind (Noebauer et al., 2010; Puebla et al., 2011). Here we explore these efforts and point out the potential solutions to the problems found. Special attention is focused on the new methods for spectral synthesis and for structure calculation, comparing with the standard models.

The structure of this chapter is as follows: The following section describes the disk formation in CVs as well as the main spectral region of its radiative emission. Section 3 describes the main spectral characteristics in UV of the brightest non-magnetic CVs (NLs and DNe in outburst). Section 4 delineates the main spectral synthesis method currently used to describe the UV spectra of CVs disk, the disk-atmosphere. We make a special focus on works of Hubeny (1990); Kriz \& Hubeny (1986); Wade \& Hubeny (1998) and Puebla et al. (2007). In section 5 we describe disk wind models with a comparative analysis with UV data, specially focusing on works of Long \& Knigge (2002); Noebauer et al. (2010) and Puebla et al. (2011). Finally in section 6 we present a brief view the current stage on the spectral synthesis methods and the challenges for the future, as well as possible solutions to the problems that still remain.

\section{Disk formation and its spectrum}

The VCs are formed from binary systems with an original orbital period between 1-10 years. After the system has lost sufficient angular momentum, the secondary star can fill its Roche lobe. At this point in the evolution the mass transfer starts through a stream that falls into the primary potential well. Due to the angular momentum conservation, the stream has a velocity perpendicular to the axis that joins the stars $v_{\perp} \sim b_{1} \omega$, where $\omega=2 \pi / P_{\text {orb }}$ and $b_{1}$ is the distance between the WD and the inner Lagrange point $\left(\mathrm{L}_{1}\right.$, see figure 1) (Frank et al., 2002; Warner, 1995). For typical values for $P_{\text {orb }}$ of $C V s, v_{\perp} \sim 100 \mathrm{~km} \mathrm{~s}^{-1}$, which indicates a super-sonic flow, the streamline will orbit the WD in an elliptical trajectory. Due to its continuum flux, the stream will collide with itself. These shocks will cause energy dissipation and the circularization of the trajectory, thus forming a ring around the white dwarf. As matter accumulates within the ring, the internal friction becomes important, and viscosity processes begin to act. These processes convert the gravitational energy to internal energy, which is then radiated. This loss of energy makes the particles occupy trajectories closer to the WD. As the scale of time of this process is large when compared to radiation and dynamic time scales, the orbits of particles thus formed are almost circular. As the angular momentum is conserved, the angular momentum of the falling particles must be transported to external regions. Thus 


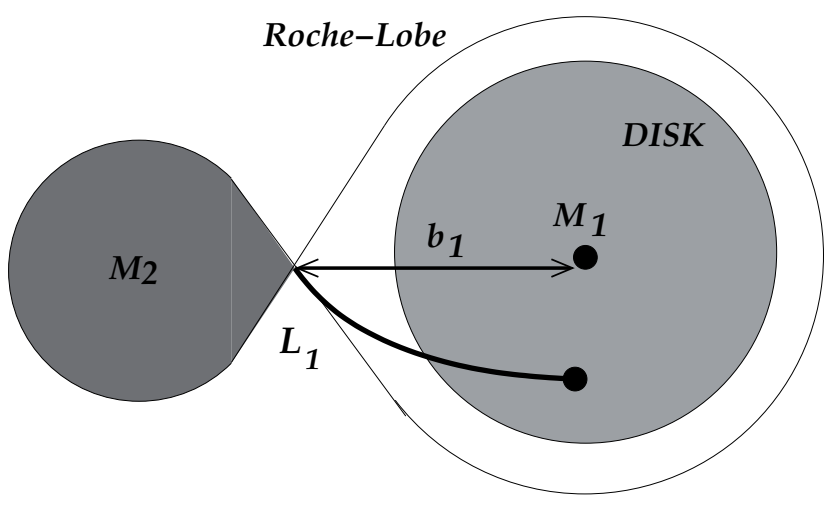

Fig. 1. Binary geometry. It shows the Roche-lobe filling secondary, accretion disk, WD (primary) and the streamline that leaves the secondary through the inner Lagrange point $\left(\mathrm{L}_{1}\right)$. It also shows the hot spot.

a small portion of gas is transported away from the white dwarf. The structure formed in this way is known as accretion disk (Frank et al., 2002).

As was mentioned above, the particle trajectories within the disk are almost circular and keplerian, balancing gravitational and centrifugal forces. Thus, the velocity will be given by:

$$
v_{K}=\sqrt{\frac{G M_{W D}}{r}}
$$

where $r$ is the distance to the WD center and $G$ the gravitational constant. It is easy to estimate the accretion luminosity of the disk if we suppose that the falling matter reaches the WD surface with the corresponding keplerian velocity. From there the disk luminosity is given by:

$$
L_{d}=\frac{\mathrm{GM}_{\mathrm{WD}} \dot{M}_{a}}{2 \mathrm{R}_{\mathrm{WD}}}=\frac{1}{2} L_{a c}
$$

where $\dot{\mathrm{M}}_{a}$ is the mass accretion rate through the disk and $\mathrm{L}_{a c}$ is the accretion luminosity. For typical values of $\dot{\mathrm{M}}_{a}$ for bright CVs $\left(10^{-10}-10^{-8} \mathrm{M}_{\odot} \mathrm{yr}^{-1}\right.$ (Puebla et al., 2007)), the disk luminosity is between $\sim 1-100$ solar luminosities $\left(\mathrm{L}_{\odot}\right)$.

One of the main problems of the accretion disk physics is to understand the origin of the viscosity that dissipates the energy and transports the angular momentum to the outer radii. Nowadays, it is accepted that the viscosity is driven by what is called magnetohydrodymacis turbulence (Balbus \& Hawley, 1991). However, some properties of the disk can be studied by using the standard Shakura \& Sunyaev (1973) $\alpha$ parametrization for kinematic viscosity $\bar{w}$ :

$$
\bar{w}=\alpha c_{S} H
$$




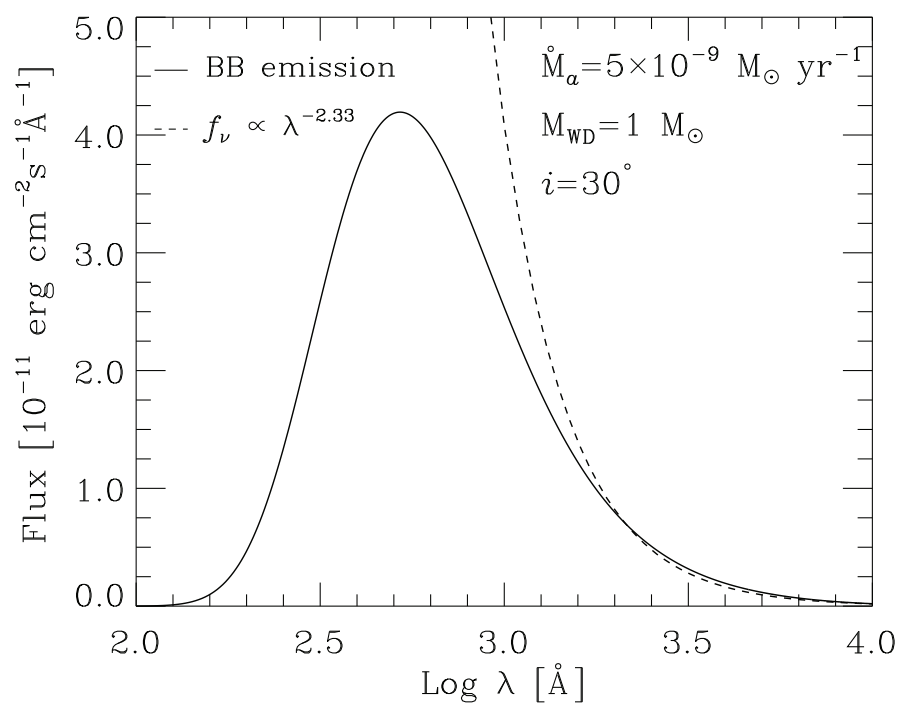

Fig. 2. The spectrum of an assemble of black bodies radiating concentric rings. The temperature of rings follows the steady model (eq.4). The parameters of system are shown in the figure. The dashed line shows the Lynden-Bell (1969) emission of an infinite disk: $f_{\lambda} \propto \lambda^{-2.33}$.

where $c_{S}$ is the sound velocity and $H$ is the hydrostatic vertical scale of the disk. In the case of a bright disk, its high mass accretion rate makes it stable (Lasota, 2001) and if we suppose that the keplerian orbits are maintained as far as a region close to the WD, the radial distribution of energy dissipated by a disk is independent of viscosity. Therefore, the effective temperature within the standard model for a steady accretion disk can be expressed by (Pringle, 1981; Shakura \& Sunyaev, 1973):

$$
T(r)=\left(\frac{3 G \mathrm{M}_{\mathrm{WD}} \dot{M}_{a}}{8 \pi \sigma \mathrm{R}_{\mathrm{WD}}{ }^{3}}\right)^{1 / 4}\left(\frac{\mathrm{R}_{\mathrm{WD}}}{r}\right)^{3 / 4}\left[1-\left(\frac{\mathrm{R}_{\mathrm{WD}}}{r}\right)^{1 / 2}\right]^{1 / 4},
$$

where $\sigma$ is the Stefan-Boltzmann constant. If we call $\mathrm{T}_{*}=\left(3 \mathrm{GM}_{\mathrm{WD}} \dot{M}_{a} / 8 \pi \sigma \mathrm{R}_{\mathrm{WD}}{ }^{3}\right)^{1 / 4}$, the temperature maximum is $\mathrm{T}_{\max }=0.49 \mathrm{~T}_{*}$ at $r=(49 / 36) \mathrm{R}_{\mathrm{WD}}$. Thus, for the typical physical parameters for luminous VCs, the accretion disk have temperatures between $50000 \mathrm{~K}$ and $10000 \mathrm{~K}$. Therefore, the disk spectrum is mainly emitted in UV between 500-3000 $\AA$, hence the importance of the spectroscopy in this spectral region to understand the accretion disk physics. For a disk formed by an assembling of infinite black body emitting rings, Lynden-Bell (1969) shows that the spectrum has the form: $f_{\lambda} \propto \lambda^{-2.33}$. Figure 2 shows the spectrum of a finite accretion disk, also as an assembling of blackbody emitting rings with an accretion mass rate $\dot{\mathrm{M}}_{a}=5 \times 10^{-9} \mathrm{M}_{\odot} \mathrm{yr}^{-1}$, a $\mathrm{M}_{W D}=1 \mathrm{M}_{\odot}$ and an orbital inclination $i=30^{\circ}$. The external radius of disk $\left(r_{\text {disk }}\right)$ is set through the tidal forces that disrupt the disk to $\sim 0.8 \mathrm{~b}_{1}$ (Osaki et al., 1993). Figure 2 also shows the disk spectrum from Lynden-Bell (1969) $f_{\lambda} \propto \lambda^{-2.33}$ (dashed line), which has a good similarity at low wavelengths (optical to middle ultraviolet, MUV), but a large discrepancy at short wavelengths (far, FUV, to extreme, EUV ultraviolet). It is clear that 
for this kind of accretion disk, the emission peak falls on UV for typical parameters $\left(\mathrm{M}_{\mathrm{WD}}\right.$ and $\dot{\mathrm{M}}_{a}$ ) found for CVs.

\section{UV spectroscopy of non-magnetic CVs}

For nearly 30 years the UV emission from CVs has been deeply studied through data taken form satellites (e.g. IUE, HST, HUT and FUSE). These studies have helped to constrain the main physical characteristics of disks. In general, the UV spectra of non-magnetic CVs show a strongly blue continuum and strong lines of high ionization as: C IV, N V, Si IV and He II. However, absorption and emission lines of an intermediately-to-low ionization state were also observed, as C III, C II, Si III, Si II and others. These features would show a strongly stratified temperature and density structure that would produce a complex ionization distribution. Here, we will focus on the spectral features of high state accretion disks, namely, NLs and DNe in outburst.

\subsection{Continuum}

For several decades a variety of models have been developed to reproduce the photometric and spectroscopic data. For the last case, the first efforts were made performing models with an arrange of concentric rings that radiate as black-bodies following the standard temperature radial distribution (eq. 4). This models bears a UV continuum that follows the $f_{\lambda} \propto \lambda^{-2.33}$ form for an infinite disk (Lynden-Bell, 1969) or for a finite disk as was shown in figure 2 (Lynden-Bell \& Pringle, 1974; Pringle, 1981; Tylenda, 1977). The first attempts to incorporate line and continuum opacities in the calculus of UV emission was performed by La Dous (1989). In a parallel, a composite of concentric rings of Kurucz (1979) stellar atmospheres was calculated by Wade (1984). Although these models have managed to reproduce some spectral features, a crucial problem was found. Wade (1988) tried to fit these models with the UV data for nine NLs and pointed out that when the models match the flux level cannot match the spectrum slope. This problem is now know as the "color-magnitude" dichotomy or the "color-magnitude" problem. Few years later, these kinds of models were followed by more realistic ones. They calculate the whole vertical structure assuming that physical properties of the disk vary in the vertical sense faster than in the radial direction. Also, the models take into account the vertical dependence of gravity and an internal energy generation by viscosity (Kriz \& Hubeny, 1986; Shaviv \& Wehrse, 1986). The internal viscosity is parameterized through algebraic expressions compatible with the $\alpha$ models of disks (Shakura \& Sunyaev, 1973). Besides, in these models, the following are taken into account: line blanketing, Doppler broadening and limb darkening (Diaz et al., 1996; Wade \& Hubeny, 1998). These models are known as: "disk atmospheres". In the subsequent years, the WD's emission, orbital phase and disk rim influence on model spectrum were included (Linnell et al., 2007; Linnell \& Hubeny, 1996).

This generation of models is capable to reproduce some photospheric spectral features as well as the broadening of lines due to disk rotation, however some problems still persist. The main of them is the color-magnitude dichotomy found in subsequent works that used this model to study the disk properties and to estimate systems parameters (Diaz \& Hubeny, 1999; Engle \& Sion, 2005; Linnell et al., 2008; Long et al., 1994; Nadalin \& Sion, 2001). Recently, Puebla et al. (2007) used the model to fit the continuum UV for 33 CVs (NLs and old Ne) using a multi-parametric optimization method. They confirmed the same problem for the most of 


\begin{tabular}{lc|lc}
\hline \hline Ion & $\lambda(\AA)$ & Ion & $\lambda(\AA)$ \\
\hline Ly $_{\beta}$ & 1025.18 & C II & 1335.3 \\
O IV & 1031.6 & O IV & 1338.6 \\
Si IV & 1062.6 & P III & 1341.6 \\
Si IV & 1073.6 & O V & 1371 \\
P IV & 1117.8 & Si IV & $1393.8,1402.7$ \\
P V & 1128.01 & Ni IV] & 1486 \\
C III & 1175 & Si II & $1526.7,1533.4$ \\
Si III & 1201.3 & C IV & $1548.2,1550,7$ \\
Si III & 1206.5 & Mg V & 1575.2 \\
Ly $\alpha$ & 1215.67 & Fe II & 1608 \\
N V & $1238.40,1242.78$ & He II & 1640.4 \\
Si II & 1251 & N IV & 1718.5 \\
Si II & 1260,1264 & Si II & 1815 \\
Si III & $1298.9,1304.3$ & Al III & 1860 \\
[Mg V] & 1324 & Si III & 1896 \\
C II & 1323.9 & C III] & 1909 \\
\hline
\end{tabular}

Table 1. Commonly observed lines in VC's UV spectra. The wavelengths are in the rest frame.

their sample. Also, they didn't find a strong correlation between the orbital period $\left(P_{\text {orb }}\right)$ and the mass accretion rate, as is predicted by the standard evolution models and as was found by Patterson (1984).

\subsection{Lines}

One of most important characteristic of the observed UV spectra of CVs are the behavior of the line profiles. The main lines that are found in the UV spectra of CVs are shown in the table 1 with their wavelengths in rest frame. In the case of non-magnetic CVs in high state, there exists line characteristics strongly defined. The emission line are strongly dependent on orbital inclination, namely, high inclination systems show strong emission lines. Unlike them, low inclination systems show spectra mainly in absorption (La Dous, 1991). Furthermore, Puebla et al. (2007) found that low accretion rate systems don't show strong emission lines. Also, intermediate-to-low inclination systems show P Cyg profiles and/or blueshifted deep absorptions. Some examples of these characteristics are shown in figure 3 where the UV spectra of three VCs are shown, the NLs V3885 Sgr and RW Sex, and the DN in outburst Z Cam $\left(i=50^{\circ}, 35^{\circ}\right.$ and $60^{\circ}$ respectively). The spectra show strong P Cyg profiles in C IV $\lambda \lambda 1548,1551$ and deep absorptions in the rest of the lines, which are shown blueshifted. In other systems lines Si IV $\lambda \lambda 1393,1402$ and N V $\lambda \lambda 1238,1242$ also show P Cyg profiles. The absorption component of these profiles commonly shows a blue wing between 3000 to 5000 $\mathrm{km} \mathrm{s}^{-1}$ from the rest frame, and the red wing of the emission component could attain $\sim 1000$ $\mathrm{km} \mathrm{s}^{-1}$.

These features couldn't be reproduced by any model using arrays of photospheres. Reproducing the line profiles has been a hard challenge in study of accretion disk through decades. Since models using optically thin layers (e.g. Tylenda, 1981; Williams \& Ferguson, 1982) some physical frameworks have been suggested to explain the line profiles, at the present it is accepted that the these spectral structures are formed in a wind that escapes from the disk. This hypothesis is based on the correlations found between several geometrical and 


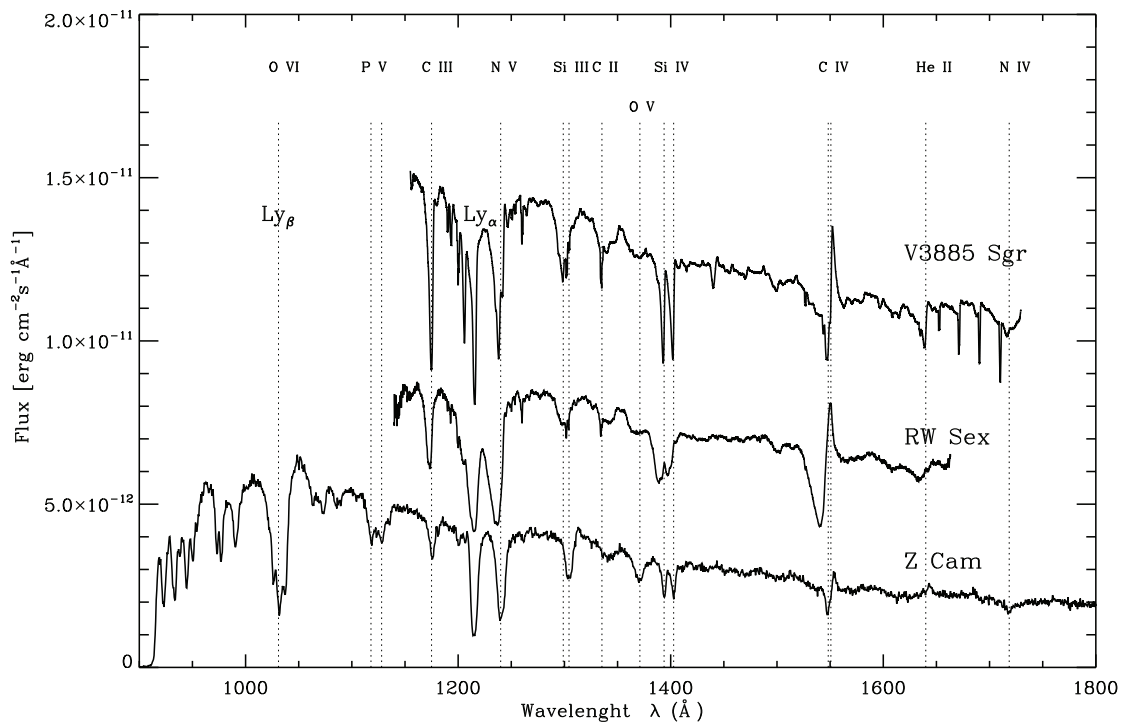

Fig. 3. UV observed spectra for three non-magnetic CVs: the NLs V3885 Sgr and RW Sex, observed by HST and the DN Z Cam observed by HUT.

physical parameters with line profiles (e.g. Shlosman \& Vitello, 1993) and the resemblance of line profiles to those observed in radiative driven winds from OB stars (Cordova \& Mason, 1982; 1985), specially the similarity of P Cyg profiles. However, there are characteristics of line profiles that are different from those ones of OB stars. For example, the minimum of flux in the absorption component of the P Cyg is close to the rest frame wavelength, unlike what happens in OB stars where it is close to the wind terminal velocity.

\section{Accretion disk atmosphere}

\subsection{The model}

In the last fifteen years, the most used method to study the UV emission of non-magnetic CVs has been the one developed by Hubeny et al. (Hubeny, 1990; Kriz \& Hubeny, 1986; Wade \& Hubeny, 1998). Here, we briefly describe the basis of this model, whose structure calculus is based on the method by Hubeny \& Lanz (1995) and its spectral synthesis on the one by Hubeny et al. (1994).

The model separates the disk in concentric rings rotating with keplerian velocity. For each ring the internal structure is calculated taking into account the internal energy generation due to viscous friction between two regions with different rotational rate (eq. 1). For the structure calculus a plane-parallel geometry is adopted. The viscous mechanical energy generated per unit volume per unit time inside the disk can be described according to Lynden-Bell \& Pringle (1974) (see also Frank et al., 2002) as:

$$
d_{m e c}=w_{l} \rho\left(r \frac{\partial \Omega}{\partial r}\right)^{2}
$$


where $w_{l}$ is the local kinematic viscosity, $\Omega$ is the angular velocity at radius $r$ and $\rho$ is the local mass density. The vertical coordinate $z$ is changed through a new parametrization by:

$$
m(z)=\int_{z}^{\infty} \rho d z^{\prime}
$$

Thus, $m(0)=M$ and $m(\infty)=0$, where $M=\Sigma / 2$, and $\Sigma(r)$ is the column mass density of disk at $r$ in $\mathrm{gr} \cdot \mathrm{cm}^{-2}$. In the model, the local viscosity $w_{l}$ is parametrized through a power function of $m$ by:

$$
w_{l}=\bar{w}(\zeta+1)\left(\frac{m}{M}\right)^{\zeta}
$$

where $\bar{w}$ is the main kinetic viscosity defined in equation (3). This expression evaluates where, within the disc, the viscous energy is released. For the structure calculus, the condition of energy balance is imposed. This condition involves that the viscous dissipation energy is equal to the radiatively released energy:

$$
\begin{aligned}
d_{\text {rad }} & =d_{\text {mech }} \\
\int_{0}^{\infty}\left(\chi_{v} S_{v}-\chi_{v} J_{v}\right) d v & =\frac{9}{16 \pi} \bar{w}(\zeta+1)\left(\frac{m}{M}\right)^{\zeta} \rho \frac{G^{\mathrm{WD}}}{r^{3}},
\end{aligned}
$$

where $\chi_{v}$ is the opacity, $S_{v}$ is the fource function and $J_{v}$ is the mean specific intensity for each frequency $v$. This will cause the non-conservative of radiation flux through the disk atmosphere, thus:

$$
\frac{d H}{d z}=4 \pi \frac{d F}{d z}=\frac{9}{16 \pi} \bar{w}(\zeta+1)\left(\frac{m}{M}\right)^{\zeta} \rho \frac{G M_{\mathrm{WD}}}{r^{3}} .
$$

This characteristic is different from the case of stellar atmospheres, where the radiative equilibrium bears a conservative flux through the structure $(d F / d z=0)$.

Another important difference with the stellar case is the gravity. In the stellar case the gravity is constant in the atmosphere, for disks the gravity is dependent on the depth. In this approximation it is not considered the self-gravity, so that gravity comes from the WD. The vertical component in this case is:

$$
g_{z}=\frac{G M_{W D}}{\left(r^{2}+z^{2}\right)^{3 / 2}} z \approx \frac{G M_{W D}}{r^{3}} z \text { for } z \ll r .
$$

With these conditions the equations for the structure are solved. These equations include the hydrostatic equilibrium, the equation of state taking into account the opacities of Hydrogen and Helium for radiation pressure, the radiative transfer equation, the radiative equilibrium equation and the statistical equilibrium equations for the level populations. The method used to solve this system of equations was developed by Hubeny (1988) and it is based on the complete linearization by Auer \& Mihalas (1969). Once the convergence is attained, the specific intensities and the flux that are emited by each ring is calculated using the code SYNSPEC $^{1}$. Then the emissions of all rings are corrected for disk rotation and integrated in the solid angle subtended by disk and the observer, taking into account the orbital inclination through (Wade \& Hubeny, 1998):

\footnotetext{
1 http://nova.astro.umd.edu
} 


$$
f_{v}=\frac{\mu}{d^{2}} \int_{r_{\min }}^{r_{\max }} \int_{0}^{2 \pi} \int_{0}^{\infty} I_{\nu}\left(\lambda^{\prime}, r, \mu\right) \Delta d r d \phi d \lambda
$$

where:

$$
\Delta=\delta\left\{\lambda-\lambda^{\prime}\left[1+\frac{1}{c}\left(\frac{\mathrm{GM}_{\mathrm{WD}}}{r}\right)^{1 / 2} \sin i \sin \phi\right]\right\}
$$

\subsection{Results}

Having a reliable of accretion disk model is crucial for understanding not only the disk physics, but also the binary system evolution. The latter due to the dependency of disk emission mainly on $\mathrm{M}_{\mathrm{WD}}$ and $\dot{\mathrm{M}}_{a}$, orbital inclination (i) and distance (d). The $\mathrm{M}_{\mathrm{WD}}, i$ and $d$ can be measured through independent methods, specially for eclipsing systems. Thus, the accretion rate, determinant in evolution models, can be obtained from disk emission.

The Hubeny's models described above, have been widely used to estimate the binary parameters or to test the model itself. This was done using first the IUE data and then, with a better resolution and signal to noise ratio, using the HST and FUSE data for FUV. Recently, we have taken the UV IUE and HST data for a sample of 33 non-magnetic CVs (10 old Ne and $23 \mathrm{NLs}$ ). We seek both, to calculate the $\dot{\mathrm{M}}_{a}$ for the sample usign the same method and to test the accretion disk emission model by Hubeny widely used now today. We used a multiparametric optimization method leaving three free parameters: $\mathrm{M}_{\mathrm{WD}}, i$ and $\dot{\mathrm{M}}_{a}$, and fixing the $d$. Thus, using this three-dimensional space of parameters we generated a set of models within it. The models were calculated between reliable ranges for free parameters. These ranges were obtained from the measured values that we found in literature. Also we collected from literature the values of $d$, the interstellar extinction parameter $E(B-V)$, and the orbital period $P_{\text {orb }}$ (Puebla et al., 2007).

The optimization was done through the minimization of the reduced $\chi_{\text {red }}^{2}$ function:

$$
\chi_{\text {red }}^{2}=\frac{1}{N-L} \sum_{i=1}^{N}\left(\frac{f_{\text {obs }}\left(\lambda_{i}\right)-f_{\text {mod }}\left(\lambda_{i}\right)}{\sigma_{i}}\right)^{2},
$$

where $N$ is the number of observed wavelengths, $L$ is the number of free parameters of the model, $f_{o b s}$ and $f_{\text {mod }}$ are the fluxes of spectra observed and modeled, respectively in the observed wavelength $\lambda_{i}$ and $\sigma_{i}$ is the error for each measured flux $f_{o b s}\left(\lambda_{i}\right)$. For the $\chi_{\text {red }}^{2}$ calculus all the lines are masked, thereby, only the continuum is taken into account for the fit. The $\chi_{\text {red }}^{2}$ values for each model thus calculated, were fitted to a smooth surface and its minimum was searched within the three-dimensional parameter space. We used a surface like the one shown in figure 4 , taking slices that cut the 3D surface of $\chi_{\text {red }}^{2}$ in some parameter. Also we calculated the influence that WD emission would have on UV. For this, we calculated the UV spectrum of a WD with the same mass and a effective temperature equal to $40000 \mathrm{~K}$. We compared the UV integrated flux of this model with the flux from the disk model. We defined $\xi$ as the ratio of these fluxes: $\xi=f_{W D} / f_{\text {disk }}$. If $\xi<0.1$ we called this models as disk dominated systems. For the system with $\xi>0.1$, we cannot conclude if the spectrum is disk dominated or not, because of the uncertainty of the real value of effective temperature. However, we found 


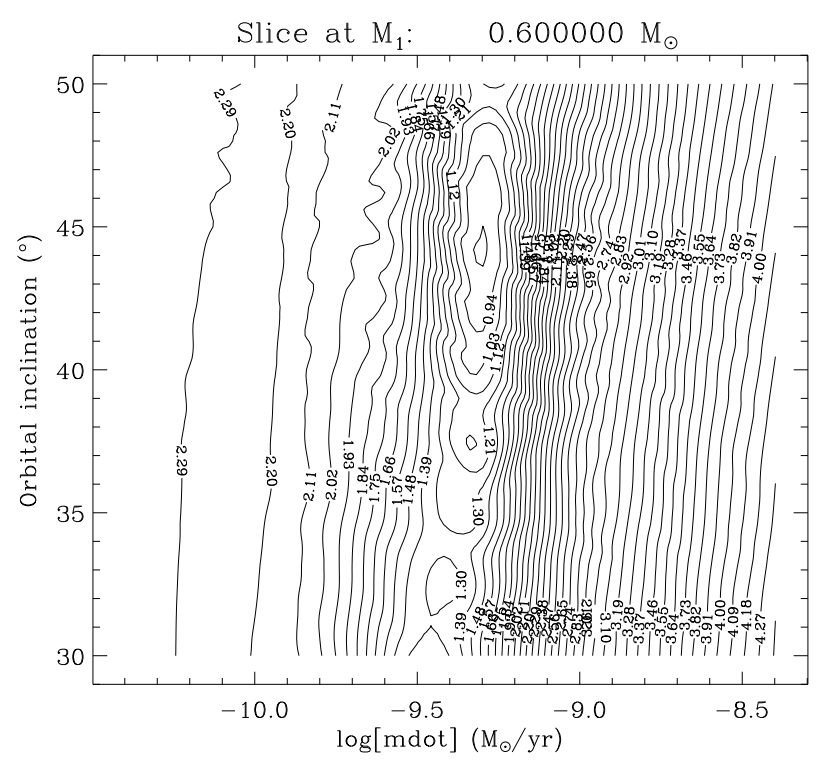

Fig. 4. Sample of a $\log \chi_{r e d}^{2}$ surface for disk spectrum fitting. This sample corresponds to the fitting of the UV spectrum of KR Aur. Figure taken from Puebla et al. (2007). Reproduced by permission of the $\mathrm{AAS}^{1}$.

that the influence of the WD emission on UV spectrum would be less than $10 \%$ for most of the systems of our sample.

In figure 4 it is shown a cut at $\mathrm{M}_{W D}=0.6 \mathrm{M}_{\odot}$ slice in the plane $\left(\dot{\mathrm{M}}_{a}, i\right)$. This figure shows an important feature of the $\chi_{\text {red }}^{2}$ surfaces. It is no possible to find an absolute minimum. The surface shows a band of multiple relative minima slightly tilted towards higher $\dot{\mathrm{M}}_{a}$ values. For higher inclination cases the slope of this band decreases due to the combined effect of aspect factor cos $i$ and limb darkening. The same applies in the case of slices of constant $\dot{\mathrm{M}}_{a}$ or $i$. The reason for this is when fitting only the continuum it is difficult to simultaneously constrain all the parameters. The UV continuum of CVs has not strong inflections, and the slope can be reproduced by a non-unique set of parameters. For the case shown in figure 4, a slight loss of flux caused by lower $\dot{\mathrm{M}}_{a}$ can be balanced with a slight lower orbital inclination. The same happens with the other combinations of parameters. Also, for the case of the slope of spectrum, a change caused by one parameter can be compensated by altering another. This degeneracy between parameters makes the modeling of continuum insufficient to fully characterize the disk. It is also found that the base of the band of minima decreases towards cooler disks (lower $\mathrm{M}_{\mathrm{WD}}$ and $\dot{\mathrm{M}}_{a}$ ). It tells us that the models, in the most cases, are too blue in comparison with the observed data. This confirms the dichotomy color-magnitude problem pointed out by Wade (1988) using stellar atmosphere, now with this model of disk-atmosphere.

\footnotetext{
${ }^{1}$ American Astronomical Society
} 


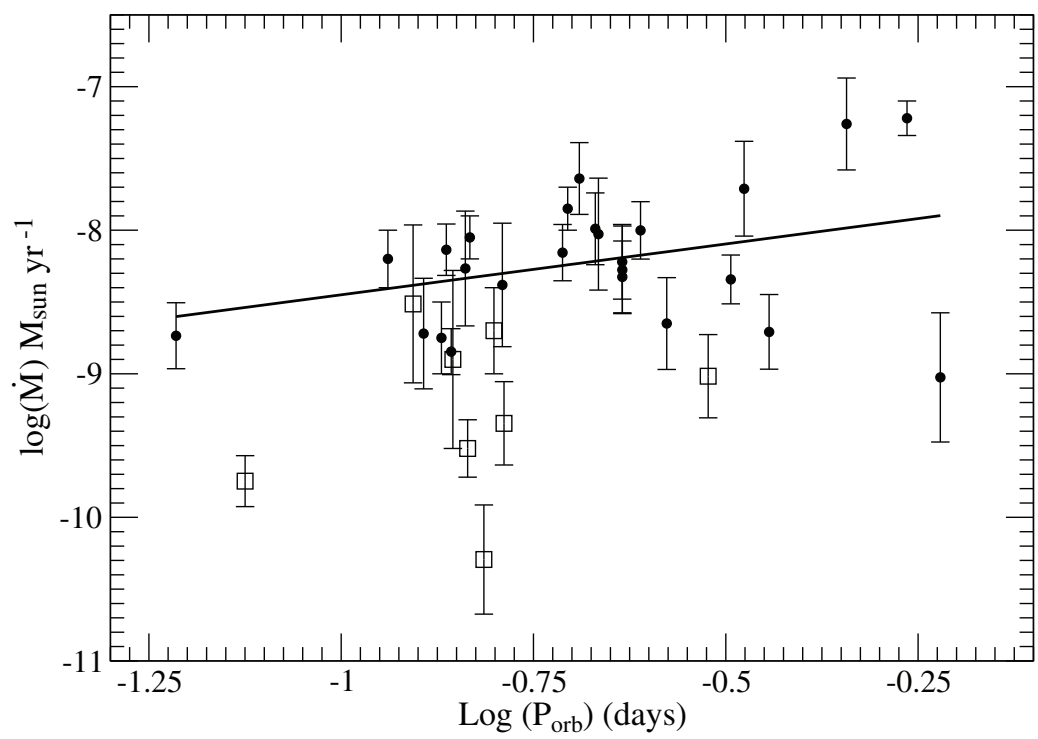

Fig. 5. Mass accretion rate $\dot{\mathrm{M}}_{a}$ as a function of orbital period. The best linear fit is shown and is expressed by equation (15). The filled circles are disk dominated CV's while the squares represents the objects with an eventual contribution from the WD. The latter ones were not considered in the linear fit. Figure taken from Puebla et al. (2007). Reproduced by permission of the AAS.

Due to the arguments exposed above, to calculate the accretion mass rate through the UV continuum emission of non-magnetic CVs, the remaining parameters must be known through independent methods. Thus, using this model we calculated the $\dot{\mathrm{M}}_{a}$ for our sample constraining the other parameters inside reliable intervals. With these values we seek correlations with others parameters of the binary aiming to find the ones predicted by evolution models.

Figure 5 shows the accretion rate as a function of the orbital period. Patterson (1984) found a strong correlation between these parameters that is predicted by the evolution models (Rappaport et al., 1983). We did not find such a strong correlation. Our values are systematically higher than Patterson's and show a higher dispersion in the $\dot{\mathrm{M}}_{a}-\mathrm{P}_{\text {orb }}$ plane. Rutten et al. (1992) analyzed a sample of six eclipsing CVs with luminous disks through the eclipse-mapping method (Horne, 1985). They also found no correlation, and their values of $\dot{\mathrm{M}}_{a}$ are more in agreement with our values (Puebla et al., 2007). The weak correlation can be expressed as shown in equation (15), where $\mathrm{P}_{\text {orb }}$ is in days. For the linear regretion the white squares from figure 5 were not taken into account, because these systems could be influenced by the WD.

$$
\begin{gathered}
\dot{M}=1.82_{-0.86}^{+1.64} \times 10^{-8} P_{\text {orb }}^{0.7 \pm 0.40} \quad \mathrm{M}_{\odot} \mathrm{yr}^{-1} \\
\dot{M}=1.69_{-0.71}^{+1.26} \times 10^{(-0.34 \pm 0.05) M_{V}-7} \quad \mathrm{M}_{\odot} \mathrm{yr}^{-1}
\end{gathered}
$$




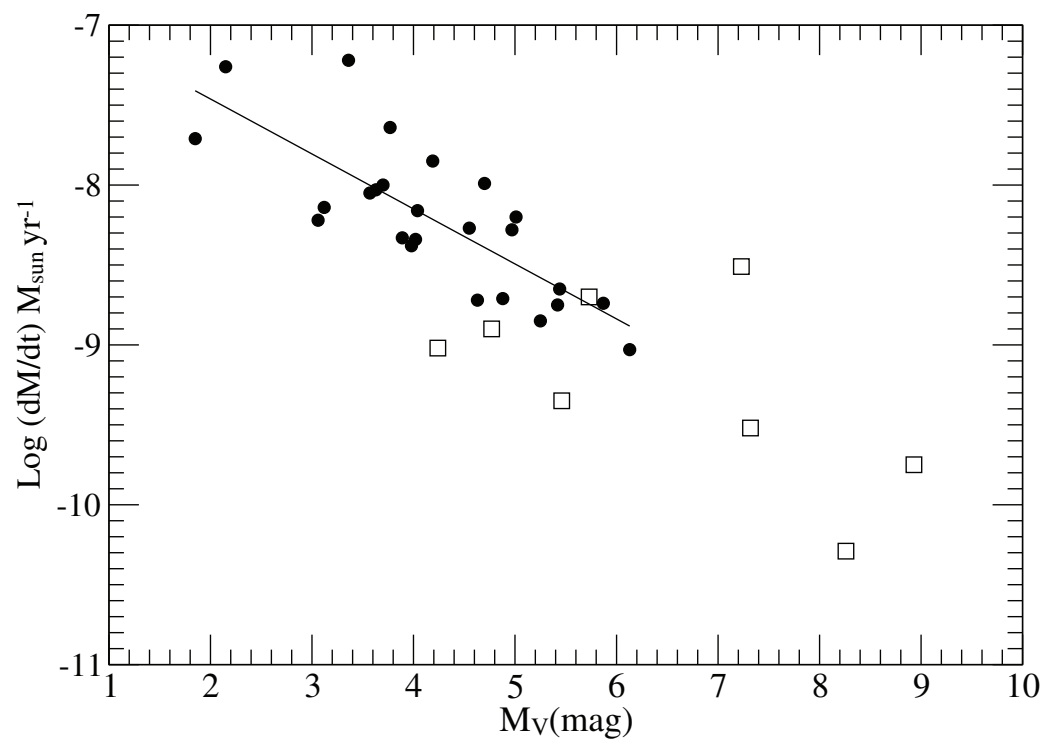

Fig. 6. Mass accretion rate $\dot{\mathrm{M}}_{a}$ as a function of the absolute magnitude of disk $\mathrm{M}_{V}$ (disk) corrected for inclination effects. The best linear fit is shown and is given by equation (16). The squares were not considered in the linear fit. Figure taken from Puebla et al. (2007). Reproduced by permission of the AAS.

Another correlation that we tried was between the absolute magnitude $\left(\mathrm{M}_{V}\right)$ and accretion rate. The absolute magnitude was corrected for inclination and limb darkening effects and calculated using the approximation of Warner (1987). Figure 6 shows $\dot{\mathrm{M}}_{a}$ as a function of $\mathrm{M}_{V}$. The correlation is evident and stronger than with the $P_{\text {orb }}$.

We also tried our optimization method setting free the distance $d$. In this case we now have a four-dimensional space for trying to simultaneously constrain the set of parameters. To let free the distance we introduced a scaling factor in order to match the flux levels. In that case the method is trying to exclusively fit the spectrum shape or slope. In the most of cases this attempt was unsuccessful, because the $\chi_{\text {red }}^{2}$ did not attain any minimum, instead the $\chi_{\text {red }}^{2}$ surfaces fall towards the redder models until living the allowed space of parameters. In a few cases we can find such kind of minima, for systems: V592 Cas, V442 Oph and CM Del. This shows that, generally, this accretion disk atmosphere model bears too blue spectra when compared with the data.

\subsection{Discussion}

The problems of this model pointed out above, don't appear with the same intensity for all binaries. Actually, each case has its own particularities. Also, there exist some cases where even this model attain to describe the UV continuum with a set of parameters compatible with the literature.

Throughout the last twenty years, some hypotheses have been proposed to overcome the problems. A widely studied hypothesis is that the standard disk model, expressed by equation 
(4), doesn't describe the real disks. Due to the fact that the model bears too blue spectra, the idea is to propose a new radial temperature structure that will yield a redder spectrum. The analysis of Rutten et al. (1992) shows that the temperature profiles of their six CVs deviate from the standard model. They show temperature profiles flatter than the steady disk. Several studies have been made to test different temperature profiles, for example Orosz \& Wade (2003) tested different temperature laws than $T(r) \propto r^{-3 / 4}$, through the law $T(r) \propto r^{-\gamma}$, with $\gamma<0.75$. They found that these profiles reduce the discrepancies between flux and color of the models. Before that, Long et al. (1994) made a detailed analysis of other possibilities in order to fit the IX Vel UV spectrum taken from HUT. They proposed three possibilities: 1) the truncation of disk at some inner disk radius, 2) setting a region of constant temperature for inner disk regions and 3) the inner disk radius could be greater than $R_{W D}$. The first possibility could be caused by a WD magnetic field disk disruption or due to inner disk evaporation (Linnell et al., 2007). In the former case, the necessary magnetic field intensity would bear a strong X-Ray emission or coherent pulsations due to the rotation of the spot of accretion, but neither of them were observed. In the case of evaporation that could be caused by the boundary layer (BL) radiation, that needs a value of mass loss that is four times higher than the predicted by the models (Linnell et al., 2007). Moreover, the BL radiation has been too hard to be detected in non-magnetic CVs. The other two possibilities can be related with assumptions made for obtaining the temperature profile (eq. 4). Specially the assumption that the keplerian disk rotation is maintained as far as the WD surface or boundary layer. This came from the presumption that $\partial \Omega / \partial r=0$ at the WD-star interface. If that condition is changed, the disk will not be keplerian everywhere and the temperature profile could be flatter. Disks without keplerian rotations appear when the complete momentum equation (including radial transport of energy) is solved, that bears a more extended boundary layer (Long et al., 1994). Another possibility is the energy that can be removed by a strong wind emerging from the inner disk regions (Linnell et al., 2007). But it is necessary too high mass loss rates to effectively act on the temperature profile (Knigge, 1999). It is almost certain that this kind of accretion disk is not totally described by the steady model (eq. 4), and new attempts are necessary to understand their structure and emission.

\section{Disk winds}

One of the most interesting challenges in the spectral synthesis of accretion disks is the UV line emission. In section 1 some spectral features were pointed that suggest that the nature of these emission profiles are a wind that is released from inner disk regions.

In the case of CVs with high accretion mass rates (NL's and DNe in eruption), the emission line profiles are strongly dependent on orbital inclination. Thus, the line strength grows with inclination (La Dous, 1991). The accretion mass rate seems to be correlated with the strength of lines. Systems with low accretion mass rate do not show strong emission lines. Figure 7 shows the strength of lines C IV $\lambda \lambda 1548,1551$ and He II $\lambda 1640$ as a function of $\dot{\mathrm{M}}_{a}$ for a sample of 33 non-magnetic CVs. It is evident that strong lines appear preferably in systems with high mass accretion rate (Puebla, 2005). Furthermore, the velocities attained by the blue wing in the P Cyg profiles show that the winds are highly accelerated. Figure 8 shows the P Cyg profiles of line C IV $\lambda \lambda 1548,1551$ for three CVs and for the O star $\zeta$ Pup. These figures show the similarity of profiles, but also some differences. For example, the minimum of depth of the blueshifted component, in the case of CVs is closer to the rest-frame wavelength, instead of $\mathrm{O}$ 


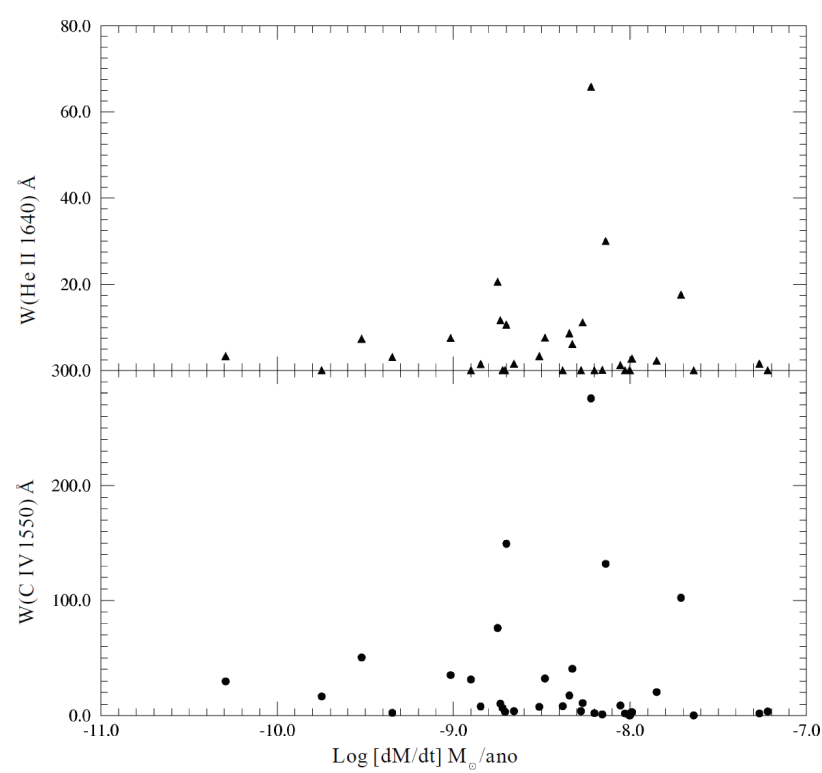

Fig. 7. Equivalent width for C IV $\lambda \lambda 1548,1551$ and He II $\lambda 1640$ as a function of accretion rate $\dot{\mathrm{M}}_{a}$. Figure taken form Puebla (2005).

stars whose minimum is commonly observed close to the terminal velocity (Prinja \& Rosen, 1995; Shlosman \& Vitello, 1993). Emission lines behavior of eclipsing systems evidences an axi-symmetric wind geometry instead of a spherical one (Mason et al., 1995). Furthermore, the multiplicity of ionization states in observed lines indicates a highly stratified ionization structure where the lines are produced. Mason et al. (1995) show that, in eclipsing systems, the lines are less eclipsed than the continuum. This suggests that the lines are produced in an extended region when compared with the origin of the continuum (the disk). There exists a correlation of the inclination not only with the strength, but also with the line profile, thus, for low-to-intermediate inclination systems P Cyg profiles are observed (e.g. DI Lac, RW Sex and HR Del), on the other hand, for systems with high inclination emission lines are seen. However, Hartley et al. (2002), from HST UV data for V3885 Sgr and IX Vel, did not find the direct correlations between the UV brightness and the wind signals as predicted by accretion disk models (Pereyra et al., 1997; 2000).

With those observational evidences, it is accepted that the winds from accretion disks are driven by line radiation, like the winds of OB stars. Efforts have been made aiming to better understand the winds from disks. Some kinds of hidrodynamical models of radiation driven disk winds have been developed in the last decades. Among them, the models of Proga et al. (1998; 1999) and Pereyra \& Kallman (2003); Pereyra et al. (1997; 2000) predict bi-polar collimated winds with high density (shock) regions and complex velocity fields. The main characteristic of such winds is that a strong stratified ionization structure is necessary in order to overcome the gravitational hill produced by the WD (eq. 11). This fact makes the disk wind notably different from the stellar winds. Specially in regard to the $\alpha$ and $k$ constants. These constants evaluate the distribution of the radiative force with the optically 


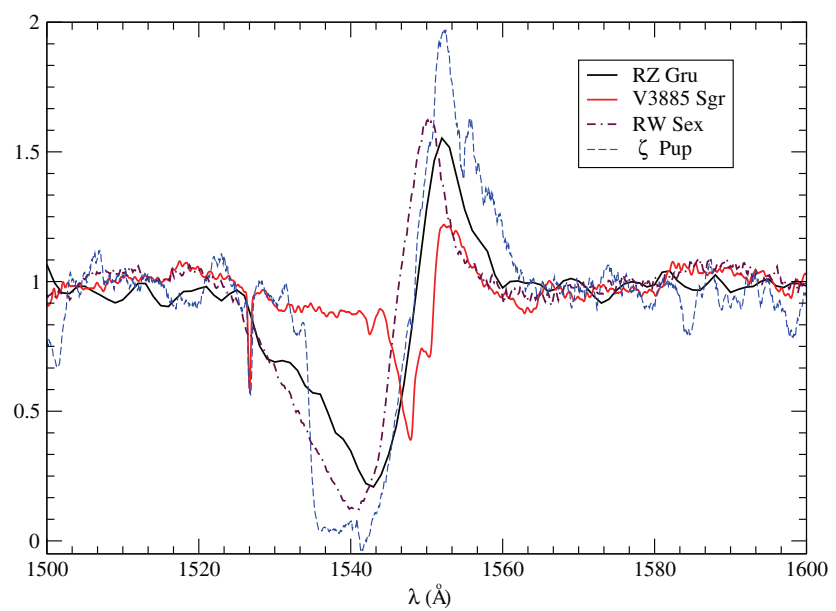

Fig. 8. Line profiles of resonant transition C IV $\lambda \lambda 1548,1551$ for the CVs RZ Gru, V3885 Sgr and RW Sex. They are compared to the IUE data for the hot O star, $\zeta$ Pup.

thin and optically thick lines taken into account to evaluate that force (Abbott, 1980; 1982). For example, Pereyra et al. (1997) concluded that it is necessary a value of $\alpha>0.5$ (many optically thick lines) to accomplish a wind from accretion disks of CVs.

Following these clues, many kinematic models and methods of spectral synthesis for disk winds have been developed. The kinematic model of Shlosman \& Vitello (1993), achieved to reproduce the emission line C IV $\lambda \lambda 1548,1551$ of RWTri, V Sge and the P Cyg profile of RW Sex. They used a stellar velocity profile and the Sobolev approximation to calculate the ionization structure. The next generation of models have used the Monte Carlo method to calculate the temperature structure consistent with the continuum emission of a steady disk (Long \& Knigge, 2002). Their work is based on the method developed by Knigge et al. (1995), that take into account the contributions of the WD, disk and eventually a BL using the exact solution for radiative transfer. They also used a stellar wind velocity profile. Long \& Knigge (2002) implemented the ionization and temperature calculus using the Sobolev approximation (Sobolev, 1957). This was the first effort to synthesize the UV spectra in a wide range of wavelengths, instead of a single line as in previous works. This method managed to reproduce well the phase behavior of the line C IV $\lambda \lambda 1548,1551$ of UX UMa (Knigge \& Drew, 1997) including a dense region in the disk-wind transition. Long (2006) pointed out how it is difficult to model all lines with the same wind parameters (collimation, temperature and ionization structure, etc.). Besides, they suggested a hybrid nature of disk wind based also on the results of Hartley et al. (2002).

Recently, we developed an alternative method to calculate the structure and spectral synthesis for accretion disk winds. In this work we tried to use a method widely employed in the study and analysis of stellar winds of hot stars. This method is based on the code CMFGEN ${ }^{1}$ (Hillier, 2003; Hillier \& Miller, 1998). We separate the disc in concentric rings and calculate a series of $1 \mathrm{D}$ models, each corresponding to a ring. For each model the inner disk, photosphere and a

\footnotetext{
${ }^{1}$ http:/ / kookaburra.phyast.pitt.edu/hillier/web/CMFGEN
} 

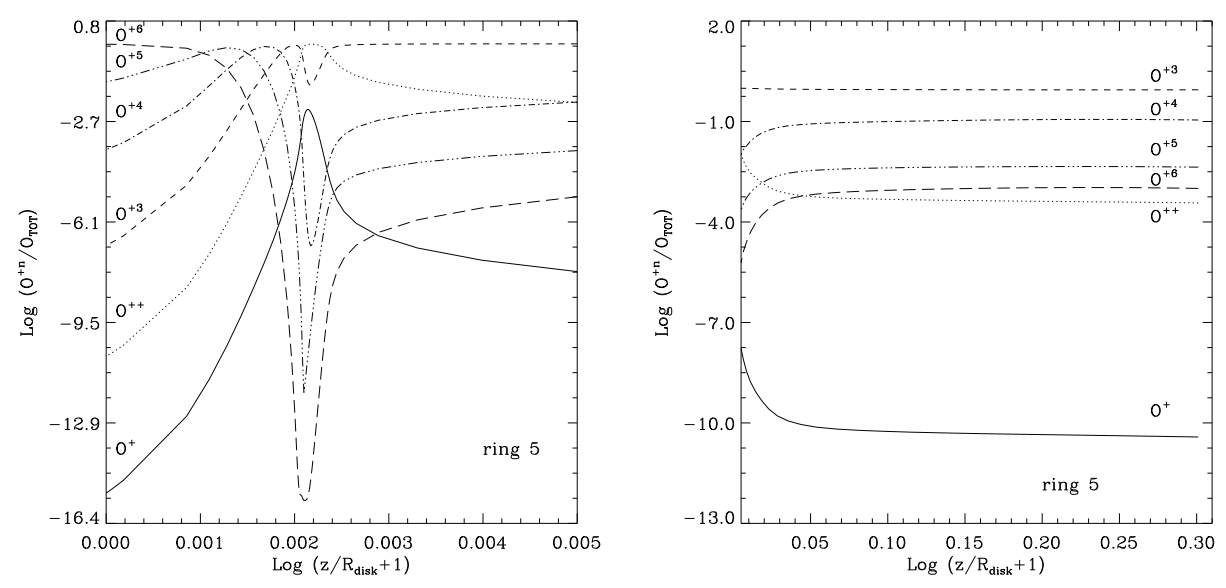

Fig. 9. Ionization structure for Oxygen of the ring 5 model (disk plus wind) with $\left(\mathrm{T}_{\text {eff }}(\mathrm{R})=38600 \mathrm{~K}\right)$. Left panel shows the inner disk and disk-wind interface. Right panel shows the extensive region of the wind.

vertical wind is calculated. The vertical density structure is calculated using a vertical velocity profile, which is computed exactly solving the Euler equation for a vertical disk wind in the context of Pereyra et al. (2004). The temperature and ionization structure is then calculated through CMFGEN in non-local thermodynamic equilibrium (NLTE) with the approximation plane-parallel. These structures show three regions: the inner and photosphere, disk-wind interface and extensive wind. Figure 9 shows the ionization structure for the oxygen for a vertical model of a ring disk with an effective temperature of $36000 \mathrm{~K}$. It is clear the strong changes in the ionization state in the disk-wind interface region (left panel) and the almost uniform structure in the extensive wind region (right panel). This kind of structures proceed from the plane-parallel approximation and has a strong influence on line profiles (Puebla et al., 2011).

The synthesis spectra follow the trends observed in the UV data of CVs. The lines ratio show a dependence on the temperature of the wind, which also depends on the accretion rate and $\mathrm{M}_{\mathrm{WD}}$. We found a dependence of the depth of absorption profiles with the $\mathrm{M}_{W D}$. In the context of the model, we found that the lower is $\mathrm{M}_{\mathrm{WD}}$, the deeper the absorption profile. Also, for low orbital inclination models we find emission profiles that are not observed, these features are caused by our simple approximation of a set of 1D plane-parallel models. However, we find that the model is capable to reproduce well the emission lines observed in high inclination systems. For RW Tri, a model with physical parameters close to those found in the literature was tested with the UV spectrum from the HST archive. Figure 10 shows two different synthetic spectra (red lines) and the HST UV data (black line). It is clear the lack of flux for the line C IV $\lambda \lambda 1548,1551$. In order to increase the flux of this line, a region of enhanced density was included between rings 1 and 4 (continuous red line). Nevertheless, it was not possible to increment the line intensity to attain the observed intensity without influencing the other lines, taking them away from the observed profiles. This shows that improvements are still necessary to the models. 


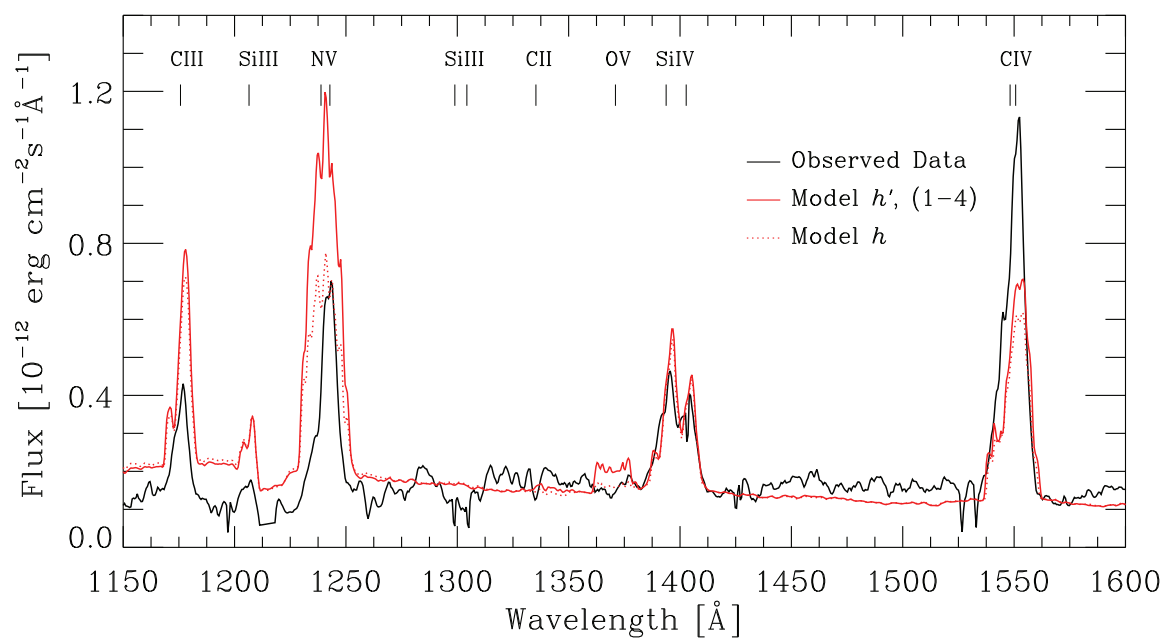

Fig. 10. Simulations for RW Tri UV (HST) data. The data are the mean of spectra taken out of eclipse. The numbers in the parentheses show the rings where a density enhancement was included. For details of $h$ and $h^{\prime}$, see Puebla et al. (2011). Figure taken from Puebla et al. (2011). Reproduced by permission of the AAS.

An advantage of this approximation is the consistent treatment of the disk-wind interface. This region is strongly influenced by the local wind acceleration. Figure 11 shows the C II $\lambda 1335$ line profile for two models with different velocity profiles (see Puebla et al., 2011, for details). The figure shows that for lines that are generated close to the disk surface the acceleration of the wind has influence on line structure as well as on line intensity. These models are still in evolution and more work is needed to improve it.

\subsection{Discussion}

The model developed by Puebla et al. (2011) has the advantage of treating consistently the disk-wind interface, but for that they sacrificed the necessary (at least) 2D treatment of the interaction between wind and disk radiation. However, they got reasonably good line profiles for high inclination models, when compared with data of the same kind of binary systems. A 2.5 dimensional (2 spacial plus rotation) treatment is necessary to improve the line profiles and to better understand the wind structure and the emission line regions in the wind.

Recently, Noebauer et al. (2010), using an improved method of Long \& Knigge (2002) (Sim et al. (2005) included the treatment of recombination lines), got good line profiles for RW Tri HST UV data. The advantage of their Monte Carlo method is the possibility of 3D treatment of radiative transfer and radiative equilibrium with a bi-conical wind geometry. They show the strong effect of the inner disk radiation on the outer and higher wind regions structures (temperature and ionization). This has a special effect on lines that are produced far from the disk, as C IV $\lambda \lambda 1548,1551$.

Data from FUSE of low inclination systems, commonly show bluehifted absorption profiles, but with low velocities $\left(\sim 100-500 \mathrm{~km} \mathrm{~s}^{-1}\right)$. These values contrast with the high velocities observed in the longward region of the spectrum. This could be a signal that a region of low accelerated wind could exist in the wind-disk interface, and that the wind could be 


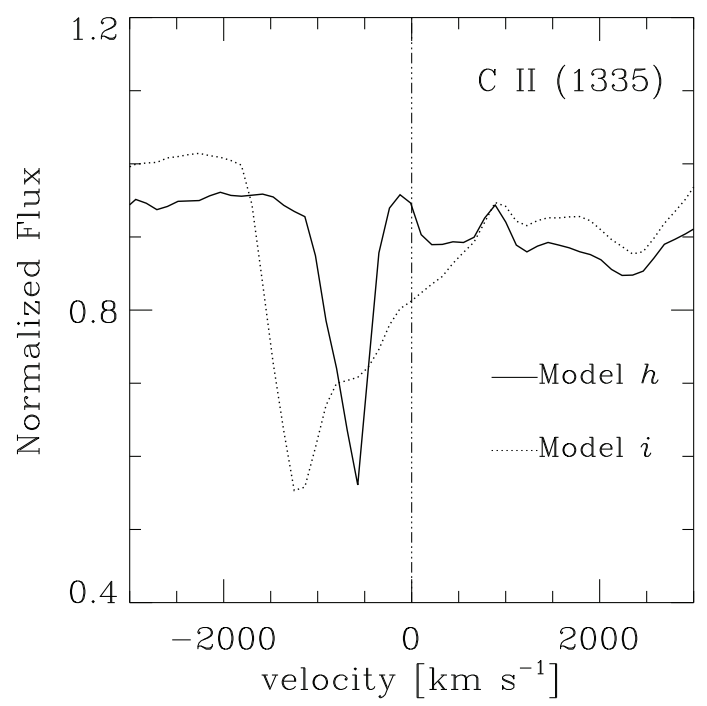

Fig. 11. C II $\lambda 1335$ line profiles for two models with different acceleration law (for details see Puebla et al. (2011)). They show the effect of the different acceleration laws on the photosphere-wind interface region. Figure taken from Puebla et al. (2011). Reproduced by permission of the AAS.

strongly accelerated in regions farther from the disk. These facts agree with the high density region postulated by Knigge \& Drew (1997) and would suggest the importance of an extended disk-wind interface. This possibility should be more explored.

\section{Conclusion and future work}

Modeling accretion disk emission is still a challenge for understanding the accretion physics. UV spectroscopy of CVs is crucial to understand the physical processes in accretion disks.

Many efforts have been done to develop models that can describe the spectroscopic data (continuum and lines). For the continuum, observational evidences show that the accretion disks don't follow the steady state disk model. This model cannot fit the color and flux at the same time using the known parameters of a system. Observational trends suggests a shallower temperature profile than the predicted by the steady model. Some alternatives to solve this problem have been raised through the years, among them: evaporation of inner disk regions, non-keplerian disks, influence of a WD magnetic field, the influence of a hot WD on the inner disk radius and accretion disk winds.

With respect to the emission line, observational clues suggest that they are formed in a wind that emerges from the disk. The complexity of the problem claims for an at least 2.5 $\mathrm{D}$ approximation calculus. Observational evidences point towards a strong influence of a disk-wind interface on spectral features. That interface was recently consistently modelated. These models show that the wind acceleration close to the disk surface strongly influences the line profile. Also, works using Monte Carlo methods have shown the importance of the inner regions on the whole wind structure, and therefore on the line profiles that are formed farther from the disk surface. 
Work is being done to improve the models described here, it is necessary to know the actual structure of disk that bears its continuum emission and the nature of the wind and the emission lines. More consistent methods are under development aiming to establish more restrictions to the physical processes in the disk.

\section{Acknowledgments}

We thank FAPESP (process: 2010/16010-7) for financial support. MPD thanks support form CNPq under grant: 305725 .

\section{References}

Abbott, D. C. (1980). The theory of radiatively driven stellar winds. I - A physical interpretation, ApJ 242: 1183-1207.

Abbott, D. C. (1982). The theory of radiatively driven stellar winds. II - The line acceleration, ApJ 259: 282-301.

Auer, L. H. \& Mihalas, D. (1969). Non-Lte Model Atmospheres. I. Radiative Equilibrium Models with - Alpha, ApJ 156: 157-+.

Balbus, S. A. \& Hawley, J. F. (1991). A powerful local shear instability in weakly magnetized disks. I - Linear analysis. II - Nonlinear evolution, ApJ 376: 214-233.

Cordova, F. A. \& Mason, K. O. (1982). High-velocity winds from a dwarf nova during outburst, ApJ 260: 716-721.

Cordova, F. A. \& Mason, K. O. (1985). High-velocity winds in close binaries with accretion disks. II - The view along the plane of the disk, ApJ 290: 671-682.

Diaz, M. P. \& Hubeny, I. (1999). The Eclipsing Cataclysmic Variable V347 Puppis Revisited, ApJ 523: 786-796.

Diaz, M. P., Wade, R. A. \& Hubeny, I. (1996). Ultraviolet Limb Darkening and Spectra for Accretion Disks in Cataclysmic Variables, ApJ 459: 236-+.

Engle, S. G. \& Sion, E. M. (2005). A Far-Ultraviolet Study of the Old Nova V841 Ophiuchi, PASP 117: 1230-1235.

Frank, J., King, A. \& Raine, D. J. (2002). Accretion Power in Astrophysics: Third Edition.

Hartley, L. E., Drew, J. E., Long, K. S., Knigge, C. \& Proga, D. (2002). Testing the line-driven disc wind model: time-resolved ultraviolet spectroscopy of IX Vel and V3885Sgr, MNRAS 332: 127-143.

Hillier, D. J. (2003). On the Solution of the Statistical Equilibrium Equations, in I. Hubeny, D. Mihalas, \& K. Werner (ed.), Stellar Atmosphere Modeling, Vol. 288 of Astronomical Society of the Pacific Conference Series, pp. 199-+.

Hillier, D. J. \& Miller, D. L. (1998). The Treatment of Non-LTE Line Blanketing in Spherically Expanding Outflows, ApJ 496: 407-+.

Horne, K. (1985). Images of accretion discs. I - The eclipse mapping method, MNRAS 213: $129-141$.

Hubeny, I. (1988). A computer program for calculating non-LTE model stellar atmospheres, Computer Physics Communications 52: 103-132.

Hubeny, I. (1990). Vertical structure of accretion disks - A simplified analytical model, ApJ 351: 632-641. 
Hubeny, I. \& Lanz, T. (1995). Non-LTE line-blanketed model atmospheres of hot stars. 1: Hybrid complete linearization/accelerated lambda iteration method, ApJ 439: 875-904.

Hubeny, I., Lanz, T. \& Jeffery, C. (1994). , St. Andrew Univ. Newsletter on Analysis of Astronomical Spectra 20: 30 .

Knigge, C. (1999). The effective temperature distribution of steady-state, mass-losing accretion discs, MNRAS 309: 409-420.

Knigge, C. \& Drew, J. E. (1997). Eclipse Mapping of the Accretion Disk Wind in the Cataclysmic Variable UX Ursae Majoris, ApJ 486: 445-+.

Knigge, C., Woods, J. A. \& Drew, J. E. (1995). The application of Monte Carlo methods to the synthesis of spectral line profiles arising from accretion disc winds, MNRAS 273: 225-248.

Kriz, S. \& Hubeny, I. (1986). Models and theoretical spectra of accretion discs in dwarf novae, Bulletin of the Astronomical Institutes of Czechoslovakia 37: 129-142.

Kurucz, R. L. (1979). Model atmospheres for G, F, A, B, and O stars, ApJS 40: 1-340.

La Dous, C. (1989). Synthetic optical and ultraviolet spectra of stationary accretion disks, $A \mathcal{E} A$ 211: 131-155.

La Dous, C. (1991). New insights from a statistical analysis of IUE spectra of dwarf novae and nova-like stars. I - Inclination effects in lines and continua, AEA 252: 100-122.

Lasota, J. (2001). The disc instability model of dwarf novae and low-mass X-ray binary transients, New A Rev. 45: 449-508.

Linnell, A. P., Godon, P., Hubeny, I., Sion, E. M. \& Szkody, P. (2007). A Synthetic Spectrum and Light-Curve Analysis of the Cataclysmic Variable IX Velorum, ApJ 662: 1204-1219.

Linnell, A. P., Godon, P., Hubeny, I., Sion, E. M. \& Szkody, P. (2008). Modeling UX Ursae Majoris: An Abundance of Challenges, ApJ 688: 568-582.

Linnell, A. P. \& Hubeny, I. (1996). A Spectrum Synthesis and Light Synthesis Program for Binary Stars with Optically Thick Accretion Disks, ApJ 471: 958-+.

Long, K. S. (2006). Far ultraviolet spectroscopy of (non-magnetic) cataclysmic variables, Advances in Space Research 38: 2827-2831.

Long, K. S. \& Knigge, C. (2002). Modeling the Spectral Signatures of Accretion Disk Winds: A New Monte Carlo Approach, ApJ 579: 725-740.

Long, K. S., Wade, R. A., Blair, W. P., Davidsen, A. F. \& Hubeny, I. (1994). Observations of the bright novalike variable IX Velorum with the Hopkins Ultraviolet Telescope, ApJ 426: 704-715.

Lynden-Bell, D. (1969). Galactic Nuclei as Collapsed Old Quasars, Nature 223: 690-694.

Lynden-Bell, D. \& Pringle, J. E. (1974). The evolution of viscous discs and the origin of the nebular variables., MNRAS 168: 603-637.

Mason, K. O., Drew, J. E., Cordova, F. A., Horne, K., Hilditch, R., Knigge, C., Lanz, T. \& Meylan, T. (1995). Eclipse observations of an accretion disc wind, MNRAS 274: 271-286.

Nadalin, I. \& Sion, E. M. (2001). The Accretion Disk and White Dwarf in the Short-Period Dwarf Novae TY Piscium and V436 Centauri during Quiescence, PASP 113: 829-834.

Noebauer, U. M., Long, K. S., Sim, S. A. \& Knigge, C. (2010). The Geometry and Ionization Structure of the Wind in the Eclipsing Nova-like Variables RW Tri and UX UMa, ApJ 719: 1932-1945. 
Orosz, J. A. \& Wade, R. A. (2003). Ultraviolet Spectra of Cataclysmic Variable Accretion Disks with Nonsteady T(r) Laws, ApJ 593: 1032-1039.

Osaki, Y., Hirose, M. \& Ichikawa, S. (1993). Tidal Effects on Accretion Disks in Close Binary Systems, pp. 272-+.

Patterson, J. (1984). The evolution of cataclysmic and low-mass X-ray binaries, ApJS 54: 443-493.

Pereyra, N. A. \& Kallman, T. R. (2003). Hydrodynamic Models of Line-driven Accretion Disk Winds. III. Local Ionization Equilibrium, ApJ 582: 984-1000.

Pereyra, N. A., Kallman, T. R. \& Blondin, J. M. (1997). Hydrodynamical Models of Line-driven Accretion Disk Winds, ApJ 477: 368-+.

Pereyra, N. A., Kallman, T. R. \& Blondin, J. M. (2000). Hydrodynamic Models of Line-driven Accretion Disk Winds. II. Adiabatic Winds from Nonisothermal Disks, ApJ 532: 563-580.

Pereyra, N. A., Owocki, S. P., Hillier, D. J. \& Turnshek, D. A. (2004). On the Steady Nature of Line-Driven Disk Winds, ApJ 608: 454-469.

Pringle, J. E. (1981). Accretion discs in astrophysics, ARAEA 19: 137-162.

Prinja, R. K. \& Rosen, R. (1995). High-resolution IUE spectroscopy of fast winds from cataclysmic variables, MNRAS 273: 461-474.

Proga, D., Stone, J. M. \& Drew, J. E. (1998). Radiation-driven winds from luminous accretion discs, MNRAS 295: 595-+.

Proga, D., Stone, J. M. \& Drew, J. E. (1999). Line-driven disc wind models with an improved line force, MNRAS 310: 476-482.

Puebla, R. E. (2005). Sobre a Taxa de Transfêrencia de Massa em Variáveis Cataclísmicas Quiescentes, Master's thesis, São Paulo: Universidade 1.

Puebla, R. E., Diaz, M. P., Hillier, D. J. \& Hubeny, I. (2011). A Method for the Study of Accretion Disk Emission in Cataclysmic Variables I: The Model, accepted in ApJ .

Puebla, R. E., Diaz, M. P. \& Hubeny, I. (2007). A Statistical Study of Accretion Disk Model Spectra for Cataclysmic Variables, AJ 134: 1923-1933.

Rappaport, S., Joss, P. C. \& Webbink, R. F. (1982). The evolution of highly compact binary stellar systems, ApJ 254: 616-640.

Rappaport, S., Verbunt, F. \& Joss, P. C. (1983). A new technique for calculations of binary stellar evolution, with application to magnetic braking, ApJ 275: 713-731.

Ritter, H. \& Kolb, U. (2003). Catalogue of cataclysmic binaries, low-mass X-ray binaries and related objects (Seventh edition), AEA 404: 301-303.

Rutten, R. G. M., van Paradijs, J. \& Tinbergen, J. (1992). Reconstruction of the accretion disk in six cataclysmic variable stars, AEA 260: 213-226.

Shakura, N. I. \& Sunyaev, R. A. (1973). Black holes in binary systems. Observational appearance., AEA 24: 337-355.

Shaviv, G. \& Wehrse, R. (1986). The vertical temperature stratification and corona formation of accretion disc atmospheres, AEA 159: L5-L7.

Shlosman, I. \& Vitello, P. (1993). Winds from accretion disks - Ultraviolet line formation in cataclysmic variables, ApJ 409: 372-386.

Sim, S. A., Drew, J. E. \& Long, K. S. (2005). Two-dimensional Monte Carlo simulations of HI line formation in massive young stellar object disc winds, MNRAS 363: 615-627.

Sobolev, V. V. (1957). The Diffusion of L $\alpha$ Radiation in Nebulae and Stellar Envelopes., Soviet Astronomy 1: 678-+. 
Tylenda, R. (1977). The continuous radiation emitted by accretion discs in cataclysmic binaries - The dwarf nova SS CYG during outburst and the old novae V 603 AQL and RR PIC, Acta Astronomica 27: 235-249.

Tylenda, R. (1981). Radiation from Optically Thin Accretion Discs, Acta Astronomica 31: 127-+.

Wade, R. A. (1984). A double grid of accretion disc model spectra for cataclysmic variable stars, MNRAS 208: 381-398.

Wade, R. A. (1988). A test of synthetic accretion disk spectra using ultraviolet flux distributions of novalike variables, ApJ 335: 394-405.

Wade, R. A. \& Hubeny, I. (1998). Detailed Mid- and Far-Ultraviolet Model Spectra for Accretion Disks in Cataclysmic Binaries, ApJ 509: 350-361.

Warner, B. (1987). Absolute magnitudes of cataclysmic variables, MNRAS 227: 23-73.

Warner, B. (1995). Cataclysmic variable stars, Cambridge Astrophysics Series, Cambridge, New York: Cambridge University Press, |c1995.

Williams, R. E. \& Ferguson, D. H. (1982). He I line emission and the helium abundance in cataclysmic variables, ApJ 257: 672-685. 


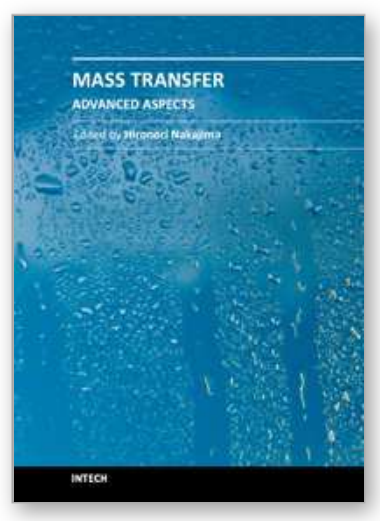

\author{
Mass Transfer - Advanced Aspects \\ Edited by Dr. Hironori Nakajima
}

ISBN 978-953-307-636-2

Hard cover, 824 pages

Publisher InTech

Published online 07, July, 2011

Published in print edition July, 2011

Our knowledge of mass transfer processes has been extended and applied to various fields of science and engineering including industrial and manufacturing processes in recent years. Since mass transfer is a primordial phenomenon, it plays a key role in the scientific researches and fields of mechanical, energy, environmental, materials, bio, and chemical engineering. In this book, energetic authors provide present advances in scientific findings and technologies, and develop new theoretical models concerning mass transfer. This book brings valuable references for researchers and engineers working in the variety of mass transfer sciences and related fields. Since the constitutive topics cover the advances in broad research areas, the topics will be mutually stimulus and informative to the researchers and engineers in different areas.

\title{
How to reference
}

In order to correctly reference this scholarly work, feel free to copy and paste the following:

Raul E. Puebla and Marcos P. Diaz (2011). UV Emission and Spectral Synthesis of Accretion Disks in NonMagnetic Cataclysmic Variables, Mass Transfer - Advanced Aspects, Dr. Hironori Nakajima (Ed.), ISBN: 978953-307-636-2, InTech, Available from: http://www.intechopen.com/books/mass-transfer-advancedaspects/uv-emission-and-spectral-synthesis-of-accretion-disks-in-non-magnetic-cataclysmic-variables

\section{INTECH}

open science | open minds

\section{InTech Europe}

University Campus STeP Ri

Slavka Krautzeka 83/A

51000 Rijeka, Croatia

Phone: +385 (51) 770447

Fax: +385 (51) 686166

www.intechopen.com

\section{InTech China}

Unit 405, Office Block, Hotel Equatorial Shanghai

No.65, Yan An Road (West), Shanghai, 200040, China

中国上海市延安西路65号上海国际贵都大饭店办公楼405单元

Phone: +86-21-62489820

Fax: $+86-21-62489821$ 
(C) 2011 The Author(s). Licensee IntechOpen. This is an open access article distributed under the terms of the Creative Commons Attribution 3.0 License, which permits unrestricted use, distribution, and reproduction in any medium, provided the original work is properly cited. 